\title{
Identification of MOS9 as an interaction partner for chalcone synthase in the nucleus
}

\author{
Jonathan I. Watkinson ${ }^{1}$ ， Peter A. Bowerman ${ }^{1}$ ， Kevin C. Crosby ${ }^{1}$ ， Sherry B. Hildreth ${ }^{1,2}$, Richard F. Helm ${ }^{2}$, \\ Brenda S.J. Winkel ${ }^{\text {Corresp. } 1}$ \\ 1 Department of Biological Sciences, Virginia Polytechnic Institute and State University (Virginia Tech), Blacksburg, VA, United States \\ 2 Department of Biochemistry, Virginia Polytechnic Institute and State University (Virginia Tech), Blacksburg, VA, United States \\ Corresponding Author: Brenda S.J. Winkel \\ Email address: winkel@vt.edu
}

Plant flavonoid metabolism has served as a platform for understanding a range of fundamental biological phenomena, including providing some of the early insights into the subcellular organization of metabolism. Evidence assembled over the past three decades points to the organization of the component enzymes as a membrane-associated complex centered on the entry-point enzyme, chalcone synthase (CHS), with flux into branch pathways controlled by competitive protein interactions. Flavonoid enzymes have also been found in the nucleus in a variety of plant species, raising the possibility of alternative or moonlighting functions for these proteins in this compartment. Here we present evidence that CHS interacts with MOS9, a nuclear-localized protein that has been linked to epigenetic control of $R$ genes that mediate effector-triggered immunity (ETI).

Overexpression of MOS9 results in a reduction of CHS transcript levels and a metabolite profile that substantially intersects with the effects of a null mutation in $\mathrm{CHS}$. These results suggest that the MOS9-CHS interaction may point to a previously-unknown mechanism for controlling the expression of the highly dynamic flavonoid pathway. 


\section{Identification of MOS9 as an interaction partner for 2 chalcone synthase in the nucleus}

3

4 Jonathan I. Watkinson ${ }^{1}$, Peter A. Bowerman ${ }^{1,3}$, Kevin C. Crosby ${ }^{1,4}$, Sherry B. Hildreth ${ }^{1,2}$, 5 Richard F. Helm ${ }^{2}$ and Brenda S.J. Winkel ${ }^{1}$

6 Departments of ${ }^{1}$ Biological Sciences and ${ }^{2}$ Biochemistry, Virginia Tech, Blacksburg, VA 24061

7

8 Current addresses: ${ }^{3}$ Division of Plant Science, BASF; ${ }^{4}$ Department of Pharmacology, University

9 of Colorado School of Medicine

10

11 Corresponding Author:

12 Brenda S.J. Winkel

13 Email address: winkel@vt.edu

14

15

16 


\section{ABSTRACT}

Plant flavonoid metabolism has served as a platform for understanding a range of fundamental biological phenomena, including providing some of the early insights into the subcellular organization of metabolism. Evidence assembled over the past three decades points to the

22 23

24 25 26 27 28

\section{BACKGROUND}

35 pathway.

\section{5} organization of the component enzymes as a membrane-associated complex centered on the entry-point enzyme, chalcone synthase (CHS), with flux into branch pathways controlled by competitive protein interactions. Flavonoid enzymes have also been found in the nucleus in a variety of plant species, raising the possibility of alternative or moonlighting functions for these proteins in this compartment. Here we present evidence that CHS interacts with MOS9, a nuclear-localized protein that has been linked to epigenetic control of $\mathrm{R}$ genes that mediate effector-triggered immunity (ETI). Overexpression of MOS9 results in a reduction of CHS transcript levels and a metabolite profile that substantially intersects with the effects of a null mutation in CHS. These results suggest that the MOS9-CHS interaction may point to a previously-unknown mechanism for controlling the expression of the highly dynamic flavonoid

Flavonoids are well known as the distinctive red, blue, and purple pigments in flowers, fruits, and vegetables that have attracted substantial interest as beneficial phytochemicals and potential pharmaceuticals (Mouradov \& Spangenberg 2014; Panche et al. 2016; Winkel-Shirley 2001). In plants, flavonoids play critical roles in reproduction and dispersal, protection from 
40 environmental stresses including UV radiation and cold, communication with other organisms,

41 and modulation of auxin transport (Gayomba et al. 2016; Stafford 1991; Winkel-Shirley 2002).

42 Flavonoids have also been linked to plant defense, both as phytotoxins that are induced in

43 response to pathogen attack, and conversely, as metabolites that are specifically repressed as part

44 of the plant immune response (Serrano et al. 2012).

45 Flavonoid biosynthesis has been extensively characterized at the biochemical, genetic,

46 and molecular levels in a wide range of plant species and, while complex, is arguably among the

47 best-understood systems of plant specialized metabolism. It has served as a highly-tractable

48 system for the exploration of fundamental genetic, biological, and biochemical phenomena,

49 including studies over the past 35 years on the organization of this pathway as an enzyme

50 complex or "metabolon" (Hrazdina \& Jensen 1992; Shih et al. 2008; Winkel-Shirley 1999;

51 Winkel 2004). The complex is characterized by relatively loose associations among its

52 component parts, which may allow for rapid disassembly and reconstitution in response to

53 cellular and environmental cues, with the distribution of flux further mediated by competition

54 between key branchpoint enzymes for association with the entry-point enzyme, chalcone

55 synthase, which appears to serve as a hub protein in this system (Burbulis \& Winkel-Shirley

56 1999; Crosby et al. 2011; Owens et al. 2008a; Owens et al. 2008b). While there has long been

57 evidence that this system is assembled at the cytoplasmic face of the endoplasmic reticulum,

58 anchored by cytochrome P450 hydroxylases, there is growing evidence for the presence of

59 flavonoid enzymes in the nucleus and perhaps other subcellular compartments, as well (reviewed

60 in Stafford 1990; Winkel 2006; Winkel 2018).

61 In the current study, we examined the possibility that CHS interacts with additional

62 proteins that may direct the cellular localization, composition, and perhaps even activity of the 
63 flavonoid enzyme complex. Use of a yeast two-hybrid screen identified a novel interactor for

64 CHS, a nuclear-localized protein identified by Xia et al. (2013) as MOS9, a regulator of $R$ genes

65 that are critical for plant defense. High resolution metabolite profiles of seedlings over-

66 expressing this protein were consistent with a model in which interaction of MOS9 with CHS

67 modifies flavonoid metabolism, either directly or indirectly. These findings suggest that the

68 flavonoid enzyme complex may be part of a larger network of interacting proteins that connect

69 the pathway's enzymes and endproducts to specific physiological functions, including plant

70 defense, through previously-unknown mechanisms.

71

\section{METHODS}

73

74 Yeast 2-hybrid assay

75 CHS and CHI were previously cloned into pBI-880 as a fusion with the GAL4 DNA-

76 binding domain (Burbulis \& Winkel-Shirley 1999). An Arabidopsis cDNA library in pBI-771

77 (Pelaz et al. 2001) was generously provided by William Crosby. Screening was performed in

78 Hf7c cells in the presence of $5 \mathrm{mM} 3$-amino-1,2,4-triazole (3-AT) as described in Kohalmi et al.

79 (1998). Two plates were screened, each containing $\sim 2 \times 10^{7}$ cells.

\section{Bioinformatics}

Homologs of MOS9 and the protein encoded by At1g56420 were identified in other

83 species using NCBI Blast and Blink. Protein sequences were aligned and a phylogenetic tree

84 was constructed using the Clustal W algorithm in the MegAlign module of Lasergene

85 (DNASTAR, Madison, WI); bootstrapping was performed using 1000 trials. Protein structural

86 comparisons were performed using 3D-Jury (http://bioinfo.pl/Meta/). Analysis and clustering of 
87 publicly-available microarray data was performed using the compendium-wide condition search

88 tools in Genevestigator (Zimmermann et al. 2004). Secondary structures for MOS9 and the

89 protein encoded by At1g56420 were predicted using the Protean module of Lasergene v. 8

90 (DNASTAR).

91

92 Plant growth conditions

Seedlings for protein interaction, RNA expression, and localization analyses were grown

on sterile medium in Nunc OmniTrays (Fisher) containing $30 \mathrm{ml}$ of $1 \mathrm{X}$ basal MS medium (Gibco)

containing $30 \mathrm{mM}(1.03 \%)$ sucrose and $0.7 \%$ Phytagel or Bacto agar (Sigma). Seeds were

sterilized and distributed on the surface of the medium as described previously (Kubasek et al.

1992). Plates were wrapped in Nescofilm and incubated at $4^{\circ} \mathrm{C}$ for $3 \mathrm{~d}$ before transferring to a growth chamber at $22^{\circ} \mathrm{C}$ under $24 \mathrm{~h}$ light $(\sim 100 \mu \mathrm{E})$.

Seedlings for metabolite profiling were grown on sterile medium in $150 \mathrm{~mm}$ x $15 \mathrm{~mm}$ petri

dishes containing $1 \mathrm{x}$ basal MS salts supplemented with $1 \%$ sucrose and $0.8 \%$ agar. Growth

conditions were as described above except that plates were incubated for $2 \mathrm{~d}$ at $4^{\circ} \mathrm{C}$ before transfer

to the growth chamber. Whole seedlings were harvested at $5 \mathrm{~d}$ post-germination, frozen in liquid nitrogen, and stored at $-80^{\circ} \mathrm{C}$ prior to extraction.

\section{T-DNA insertion mutants}

Seeds for the T-DNA insertional mutant, SAIL_622_D10, were obtained from the

Arabidopsis Biological Resource Center (ABRC). Plants homozygous for the insertion were

107 identified by PCR analysis of genomic DNA extracted from flower buds using the method of

108 Edwards et al. (1991) with the gene-specific primers to screen for intact genes and the SAIL LB1

109 and gene-specific primers to screen for T-DNA insertions (Table S1). 
111

112

113

114

115

116

117

118

119

120

121

122

123

124 125

126

127

128

129

130

131

132

\section{Plasmid constructs}

The MOS9 coding region, minus the stop codon, was amplified from clone pENTR221AT1G12530 (obtained from the ABRC, TAIR accession 1000491988) using the primers listed in Table S1. The amplified fragment was cloned into pENTR D-TOPO (Invitrogen) according to the manufacturer's instructions to generate pENTR:MOS9. This construct was recombined with pET-DEST42 (Invitrogen) using LR Clonase Plus Enzyme Mix (Invitrogen) to give MOS9::V5::6xHis for production of recombinant protein in E. coli. pENTR:MOS9 was also recombined into pEarleyGate 101 (Earley et al. 2006) using LR Clonase Plus to give de35S::MOS9::YFP-HA for stable transformation of Arabidopsis. The MOS9 construct for FLIM-FRET experiments was generated by amplifying the MOS 9 coding region using the primers listed in Table S1, which was then inserted into the BamHI site of syfp2-C1_pENTR (Crosby et al. 2011). The MOS9-syfp2 sequences were then recombined into the plant expression vector, p2BW7.0 (Karimi et al. 2002), using LR Clonase II enzyme mix (Invitrogen). The integrity of all constructs was confirmed by sequencing (performed at the Virginia Bioinformatics Institute Core Laboratory).

\section{Confirmation of protein interactions by SPR and FLIM-FRET}

Recombinant proteins were produced in E. coli BL21 (DE3) pLysS cells (Invitrogen) induced with $1 \mu \mathrm{M}$ isopropyl thiogalactopyranoside for $4 \mathrm{~h}$ at room temperature. Proteins were extracted in $10 \mathrm{mM}$ HEPES, pH7.5, $150 \mathrm{mM} \mathrm{NaCl}$, 10\% glycerol, 0.1\% Tween20, and then purified by FPLC (AKTA) over a $1 \mathrm{ml}$ nickel fast flow column (Amersham) using a linear gradient of 20-300 mM imidazole. Fractions containing the recombinant protein were combined 
133 and dialyzed against $10 \mathrm{mM}$ HEPES, $\mathrm{pH} 7.5,150 \mathrm{mM} \mathrm{NaCl}$ to remove the imidazole.

134 Immunoblot analysis of the resulting protein preparations showed that a horseradish peroxidase-

135 conjugated rabbit anti-V5 antibody (Bethel Laboratories) reacted with a band of the predicted

136 size.

137 Surface plasmon resonance refractometry (SPR) was performed on a SR7000 optical

138 biosensor (Reichert Analytical Instruments, Depew, NY) equipped with a syringe pump and

139 autosampler. Planar polyethyleneglycol/carboxyl sensor chips (Reichert) were activated with

$1400.05 \mathrm{M} \mathrm{N}$-hydroxysuccinimide, $0.2 \mathrm{M}$ and 1-ethyl-3-(3-dimethylaminopropyl) carbodiimide

141 injected at a flow rate of $5 \mu \mathrm{min}^{-1}$ for $10 \mathrm{~min}$. Recombinant CHS (1.0 $\mu \mathrm{M}$ in $10 \mathrm{mM}$ HEPES,

$142 \mathrm{pH} 7.5,150 \mathrm{mM} \mathrm{NaCl}$ containing $20 \mathrm{mM}$ sodium acetate, $\mathrm{pH} 5.0$ ) was injected over the activated

143 chip at $5 \mu 1 \mathrm{~min}^{-1}$ for $15 \mathrm{~min}$. Unbound sites were deactivated with $1 \mathrm{M}$ ethanolamine, $\mathrm{pH} 8.0$

144 for $15 \mathrm{~min}$ at $5 \mu \mathrm{min}^{-1}$ and unbound protein removed with a $10 \mathrm{~min}$ wash of $20 \mathrm{mM} \mathrm{NaOH}$ at

$14550 \mu 1 \mathrm{~min}^{-1}$. MOS9 was diluted to 0.1 to $1 \mu \mathrm{M}$ concentration in running buffer (10 mM HEPES,

$146 \mathrm{pH} 7.5,150 \mathrm{mM} \mathrm{NaCl}$ ) with $1 \mathrm{mg} / \mathrm{ml} \mathrm{BSA}$ as a blocking agent. Injections were passed over the

147 chip at $50 \mu \mathrm{lmin}-1$ for 8 min followed by 8 min dissociation in buffer without MOS9 and a $5 \mathrm{~min}$

148 wash in $20 \mathrm{mM} \mathrm{NaOH}$ to remove residual MOS9. All experiments were repeated three times

149 with the order of injections randomized. Off rate $\left(\mathrm{K}_{\mathrm{off}}\right)$ was calculated using Scrubber software

150 (version 2.0, BioLogic Software, Campbell, Australia). The $\mathrm{k}_{\mathrm{obs}}$ was calculated using Origin

151 software following a Langmuir binding plot. The association rate, $\mathrm{K}_{\mathrm{on}}$, was calculated from the

152 slope of the line plotting $k_{\text {obs }}$ vs. concentration. The affinity $\left(K_{D}\right)$ was calculated from $K_{\text {off }} / K_{\text {on }}$.

153 Frequency domain fluorescence lifetime imaging microscopy-Förster resonance energy

154 transfer (FLIM-FRET) assays were performed as described in Crosby et al. (2011). Mesophyll

155 protoplasts generated from $t t 4-11$ (Yoo et al. 2007) were transfected with the MOS9::scfp3a 
156 construct together with constructs for scfp3a, CHS::syfp2, or syfp2::CHS (Crosby et al. 2011).

157 Between 11 and 25 protoplasts were analyzed for each donor- or donor/acceptor-expressing

158 population (Table 1). FRET efficiency was calculated using lifetime values derived from phase-

159 shift, $\tau(\varphi)$, according to the formula: $E=1-\left(\tau_{\mathrm{DA}} / \tau_{\mathrm{D}}\right)$ where $\tau_{\mathrm{DA}}$ is the lifetime of the donor with

160 the acceptor presence and $\tau_{\mathrm{D}}$ is the lifetime of the donor without acceptor.

161

162 Subcellular localization

163 Transformation with the de35S::MOS9::YFP-HA construct was performed using the

164 floral dip method (Bechtold et al. 1993). Transgenic seedlings were identified by screening for

165 resistance to BASTA, followed by confirmation by PCR. T2 plants were used for localization

166 analysis. For imaging of live seedlings, 5-day old Arabidopsis were gently transferred to slides.

167 Imaging was performed using a Zeiss LSM 510 Meta confocal laser scanning microscope (Carl-

168 Zeiss, Germany) using a Zeiss apochromat 40X water objective. Samples were excited with a

$169514 \mathrm{~nm}$ argon laser. Filter sets used were a HFT 458/514 dichroic beamsplitter and a BP 530-

170600 emission filter. Images were collected in 8-bit mode with 4 times line averaging. All

171 images were collected using the same laser intensity, gain, offset, and filter settings. Stacks

172 consisting of $1 \mu \mathrm{m}$ intervals were taken to confirm the observed nuclear localization.

\section{Expression analysis and qRT-PCR}

174

RNA was extracted from roots of approximately 20, 5-day-old Arabidopsis seedlings

175 using an RNeasy RNA extraction kit (Qiagen). Each set of 20 seedlings constituted one

176 biological replicate. Biological replicates were grown on separate plates under the same

177 conditions. cDNA was synthesized using $500 \mathrm{ng}$ of total RNA from each sample as template 
178 with the MMLV Reverse transcriptase system according to the manufacturer's instruction 179 (Epicentre).

180 Quantitative real-time polymerase chain reaction (qRT-PCR) analysis was performed 181 using a 7300 Real-Time PCR System (Applied Biosystems) with SYBR Green PCR master mix 182 (Applied Biosystems). Primers were designed to assay the expression of MOS9 and CHS as well 183 as two control genes, encoding glyceraldehyde-3-phosphate dehydrogenase C-2 (GAPDH, 184 At1g13440) and ubiquitin-conjugating enzyme 21 (UBC, At5G25760). Primers were designed 185 to span introns where possible (GAPDH and $U B C$ ) to reduce the possibility of genomic 186 contamination (Table S1). Primer efficiencies were determined by linear regression of $\mathrm{Ct}$ and 187 log template concentration over a 5-log template concentration range. Efficiencies were 188 calculated using the equation: $10^{(-1 / \text { slope })}-1$. Amplification of a single target was assessed by 189 melting analysis and amplicon analysis via electrophoresis in 3\% agarose gels. Three biological 190 replicates were analyzed in triplicate using the Pfaffl $\mathrm{Ct}$ analysis method, normalizing to 191 expression of GAPDH (Pfaffl 2001). Given the possibility for variation of this control, a second 192 control gene, $U B C$, was also assayed. 


\section{Metabolite profiling}

196 Whole seedlings were ground into a fine powder by agitation of $2 \mathrm{ml}$ polypropylene tubes

197 containing the frozen tissue and stainless steel beads (2.3 mm diameter; Small Parts, Logansport, 198 IA) in a Harbil 5G paint shaker (Fluid Management, Wheeling, IL) for three 30 sec cycles. The 199 powder was extracted in 99\% methanol (Spectrum Chemicals, New Brunswick, NJ) with 1\% 200 acetic acid (Sigma, St. Louis, MO) at a concentration of $100 \mathrm{mg}$ tissue/ml, sonicated for $10 \mathrm{~min}$, 201 and then centrifuged to pellet the insoluble material. A second extraction was performed and the 202 extracts were combined. The resulting extract was dried under vacuum, reconstituted at a final 203 concentration of $50 \mathrm{mg} / \mathrm{ml}$ in $75: 25$ water:acetonitrile with $0.1 \%$ formic acid, sonicated for 10 $204 \mathrm{~min}$, and centrifuged to pellet the insoluble material.

205 Metabolite profiling was performed using a Waters Acquity I-class UPLC coupled with a 206 Waters Synapt G2-S Q-TOF mass spectrometer (Waters Corp, Milford, MA). Sample separation 207 was achieved with a binary solvent system of water (Spectrum Chemicals, New Brunswick, NJ) 208 with $0.1 \%$ formic acid (Sigma, St. Louis, MO) (A) and acetonitrile (Spectrum Chemicals, New 209 Brunswick, NJ) with $0.1 \%$ formic acid (B) with a flow rate of $0.2 \mathrm{ml} / \mathrm{min}$ using the following 210 gradient conditions: hold at 5\% B (0-1 min), linear gradient to 30\% B (1-7 min), to 95\% B (7-12

$211 \mathrm{~min})$, hold at 95\% B (12-12.5 $\mathrm{min})$, and then return to initial conditions and re-equilibrate (13-15 $212 \mathrm{~min})$. A $2 \mu \mathrm{l}$ sample was injected onto an Acquity BEH C18 column (50 mm x $2.1 \mathrm{~mm}$ i.d., 1.7 213 um; Waters Corp., Milford, MA) maintained at $40^{\circ} \mathrm{C}$.

214 The column was eluted into the mass spectrometer fitted with an ESI probe and analysis 215 was performed in high resolution, negative mode with a scan time of $0.20 \mathrm{sec}$ and a mass scan 216 range of $100-1800 \mathrm{~m} / \mathrm{z}$. Source conditions were: capillary voltage 2.4 , temperature $120^{\circ} \mathrm{C}$, cone 217 voltage 30 , source offset 80 , desolvation temperature $350^{\circ} \mathrm{C}$, cone gas $50 \mathrm{~L} / \mathrm{h}$, desolvation gas 500 
$218 \mathrm{~L} / \mathrm{h}$, and nebulizer gas 6.0 bar. For accurate mass, leucine-enkephalin (Waters Corp., Milford,

$219 \mathrm{MA}$ ) at a concentration of $200 \mathrm{ng} / \mathrm{ml}$ was continually infused through the reference sprayer at 5 $220 \mu 1 / \mathrm{min}$.

221 The metabolomic data files were processed with MarkerLynx XS application manager 222 software (version 4.1, Waters Corp., Milford, MA, USA) for unsupervised feature discovery, 223 principal component analysis (PCA), and orthogonal partial least squared discriminant analysis 224 (OPLS-DA) with processing parameters as detailed in Table S2. OPLS-DA was performed as a 225 direct comparison of the metabolomic profiles of de35S::MOS9::YFP and Col-0 to identify 226 feature differences highly correlated with the de35S::MOS9::YFP genotype. To determine the 227 metabolomic relationship of de35S::MOS9::YFP with $t t 4-11$ and mos $9-2$, the fold change and p228 value (calculated by Student's t-test) of the de35S::MOS9::YFP associated features were 229 calculated for the $t t 4-11$ and $\operatorname{mos} 9-2$ genotypes based on the MarkerLynx XS values.

230 Fragmentation analysis by MS/MS was performed to assign identities to the 231 de35S::MOS9::YFP-associated features using the same chromatography and source conditions 232 described above. Details related to fragmentation and identification of the metabolites are 233 provided in Table S3. Identification of the flavonol conjugates was performed from $\mathrm{MS}^{\mathrm{E}}$ 234 analysis, which allows for simultaneous detection of low and high collision energy data to 235 provide untargeted fragmentation information. The flavonoid conjugates were identified based 236 upon diagnostic fragment ions of the flavonols, kaempferol, quercetin, and isorhamnetin; the 237 supporting data is provided in Table S4. 


\section{RESULTS}

\section{Identification of a novel interaction partner for CHS}

Previous interaction assays suggest that CHS may serve as a hub for the assembly of a

243 flavonoid enzyme complex (Burbulis \& Winkel-Shirley 1999; Crosby et al. 2011; Owens et al.

244 2008a; Owens et al. 2008b). To survey the Arabidopsis transcriptome for other potential

245 interacting partners of CHS, a yeast two-hybrid screen was undertaken using a cDNA prey

246 library generated from whole Arabidopsis plants at different stages of development (Pelaz et al.

247 2001). Screening was performed in Hf7c cells harboring a full-length CHS bait construct in the

248 presence of $5 \mathrm{mM}$ 3-amino-1,2,4-triazole (3-AT). While a parallel experiment using a full-

249 length CHI bait construct yielded no positives, the screen with CHS yielded approximately 30

250 colonies that grew to at least $1 \mathrm{~mm}$ in diameter after $6 \mathrm{~d}$ on selective medium. Of these, six

251 exhibited robust growth after $10 \mathrm{~d}$ upon rescreening for histidine prototrophy in the presence of

252 3-AT. All six constructs tested negative for auto-activation of the His3 reporter gene in the

253 absence of the CHS bait construct. $\beta$-galactosidase activity from the secondary reporter was not

254 detected in any of the positive colonies; this was also the case in previous yeast two-hybrid

255 analyses of flavonoid enzyme interactions, suggesting that these interactions are relatively weak

256 (Burbulis \& Winkel-Shirley 1999).

257 One of the constructs contained sequences corresponding to the Arabidopsis gene,

258 At1g12530, which encoded a small (193 amino acid, $21.8 \mathrm{kD}$ ) protein of as-yet unknown

259 function. Although the coding region was out of frame with the Gal4 activation domain,

260 translational frameshifting is known to occur at high frequency in yeast (Albers et al. 2005;

261 Fromont-Racine et al. 1997). The potential interaction of the two proteins was therefore

262 examined further by SPR, using recombinant protein generated in E. coli from At1g12530 cDNA

263 as the mobile analyte against immobilized recombinant CHS. Analysis of the binding isotherms 
264 showed a good fit between $\mathrm{k}_{\mathrm{obs}}$ vs. concentration (Figure 1A) and gave a calculated $\mathrm{K}_{\mathrm{D}}$ of 210 $265 \mathrm{nM}$.

266 To determine whether the interaction between CHS and the At1g12530 gene product 267 could also be detected in vivo, the proteins were subjected to FLIM-FRET analysis (van Munster $268 \&$ Gadella 2005). The At1g12530 and CHS coding regions were fused to the optimized cyan 269 and yellow fluorescent protein variants, SCFP3A and SYFP2, respectively, and placed under 270 control of the double enhanced CaMV 35S (de35S) promoter as described previously (Crosby et 271 al. 2011). FLIM-FRET analysis in Arabidopsis mesophyll protoplasts showed no change in the 272 fluorescence lifetime of the SCFP3A-At1g12530 protein when it was expressed in the presence 273 of SYFP2 alone $(E \tau(\varphi)=0.5 \%$; Table 1). In contrast, a significant decrease in lifetime was 274 observed when this protein was expressed together with CHS-SYFP2 $(E \tau(\varphi)=6.5 \%$; Table 1; 275 Figure 1B), similar to the efficiencies previously reported for interaction with two flavonoid 276 enzymes, flavonol synthase 1 (FLS1) and dihydroflavonol 4-reductase (DFR) (Crosby et al. 277 2011). A substantially smaller effect on the lifetime of SCFP3A-At1g12530 was observed with 278 SYFP2-CHS $(\operatorname{E} \tau(\varphi)=2.8 \%$; Table 1) suggesting that fusion of the fluorescent protein to the $\mathrm{N}$ 279 terminus of CHS may interfere with the interaction or that dipole distance and/or that orientation 280 for this pair of constructs is less favorable for FRET. Position-specific effects of the fusion 281 partner were also observed in FLIM-FRET experiments for interactions between CHS, FLS1, 282 and DFR (Crosby et al. 2011). gene product interact in vivo. Remarkably, the product of this novel CHS interacting protein has 285 been identified by Xia et al. (2013) as MOS9, a 193 aa (21.8 kD calculated) protein that 286 associates with the Set1 class H3K4 methyl transferase, ATXR7, to activate transcription of 
$287 R P P 4$ and $S N C 1$ in response to pathogen effectors. Arabidopsis also contains a second related 288 gene, At1g56420, which encodes a 183 amino acid (20.4 kD calculated) hypothetical protein 289 with 32.3\% amino acid identity to MOS9 (Xia et al. 2013), which we refer to as MOS9-L; it 290 remains to be determined whether this protein has any of the same interaction partners. MOS9 291 and MOS9-L are both characterized by a high degree of predicted alpha helical structure, 292 including amphipathic alpha helices (Figure S1A). Like flavonoid enzymes, these proteins 293 appear to be unique to the green lineage, with orthologs in other plant species and smaller 294 versions (108-123 amino acids in length) encoded by the Physcomitrella, (28.6-61.1\% identity) 295 and Chlamydomonas (15.6-32.5\% identity) genomes (Figure S1B).

296

297 Co-localization and co-expression of MOS9 and CHS

CHS has been localized to both the cytoplasm, where it is to at least some extent 299 associated with the endoplasmic reticulum, and to the nucleus (Hrazdina \& Wagner 1985; 300 Saslowsky et al. 2005; Saslowsky \& Winkel-Shirley 2001). Xia et al. (2013) showed that MOS9 is located predominantly in the nucleus of root and leaf pavement cells based on analysis of GFP fusions driven by the MOS 9 promoter in transgenic plants and cell fractionation experiments. We observed a similar distribution in Arabidopsis Col-0 roots for a MOS9-YFP fusion protein expressed under control of the de35S promoter (Figure 2A and C). Predominantly nuclear localization was also the case in the absence of CHS, as evidenced from crossing one of these lines with the CHS null, tt4-11, and selecting for yellow-seeded plants expressing YFP

307 (Bowerman et al. 2012) (Figure 2B and C). The fluorescence of MOS9-YFP was considerably 308 lower in the $t t 4-11$ background, even though the absence of flavonoids in these tissues should, if 309 anything, provide for an enhanced fluorescence signal from YFP (Mercuri et al. 2001; Robic et 
310 al. 2009). The finding that MOS9 transcript levels were not statistically different in this line

311 (Figure 3A) suggests that the stability of the MOS9 protein could be enhanced upon interaction

312 with CHS.

313 The expression of MOS9 was compared to that of $C H S$ across different tissues and

314 environmental conditions using Genevestigator (Zimmermann et al. 2004). This analysis

315 indicates the both genes are expressed in almost all tissues, albeit with somewhat distinct

316 patterns of RNA accumulation (Figure S2A). Both exhibit particularly high levels of expression

317 in a variety of root and seed cell types. There are few tissues where only one of these genes is

318 expressed, notably in the hypocotyl, where MOS9 transcripts accumulate but $C H S$ does not

319 appear to be expressed. The two genes do exhibit markedly different responses to biotic and

320 abiotic stress (Figure $\mathrm{S} 2 \mathrm{~B}, \mathrm{C}$, and $\mathrm{D}$ ), with $M O S 9$ being relatively unresponsive across a range of

321 perturbations, whereas $C H S$ transcript levels change dramatically in plants exposed to diverse

322 nutritional, environmental, and biotic stresses, as has also been well documented in the literature

323 (reviewed in Mouradov \& Spangenberg 2014; Winkel-Shirley 2002). This includes significantly

$324(\mathrm{p}<0.001)$ reduced CHS transcript levels in response to several bacterial pathogens; MOS9

325 displays a modest $(-1.73$ fold; $\mathrm{p}<0.05)$ response only in leaf discs treated with the bacterial

326 flagellin peptide, flg22, and gibberellic acid (where $C H S$ is -1.91 fold, but with $\mathrm{p}=0.423$ ). Thus,

327 while $C H S$ and MOS9 have the potential to act in concert in many, if not all, cell types, only $C H S$

328 exhibits a strong stress response, including during pathogenesis.

329

330 Genetic analysis

331

Although there are no publicly-available mutant lines with disruptions of the MOS 9

332 coding region, SAIL_622_D10 contains a T-DNA insertion located $165 \mathrm{bp}$ upstream of the 
333 predicted start codon for this gene. Surprisingly, quantitative reverse transcription (qRT)-PCR

334 analysis of expression in seedling roots showed that MOS9 transcript levels, rather than being

335 diminished, were approximately 8 -fold higher in this line than in wild-type when normalized to

336 the endogenous control gene, GAPDH $(\mathrm{p}<0.02)$ (Figure $3 \mathrm{~A})$. Normalization to a second

337 endogenous control gene, $U B C$, yielded similar results (Figure $\mathrm{S} 3 \mathrm{~A}$ ). The insertion thus likely

338 disrupts a negative regulatory element in the $M O S 9$ promoter. Expression of the next closest

339 annotated gene, At 1 g12540, which is divergently transcribed from a predicted start site located 2

$340 \mathrm{~kb}$ upstream, was not affected by the insertion (Figure S3B). The insertion line was therefore

341 designated $\operatorname{mos} 9-2$. qRT-PCR also showed that MOS9-YFP transcript levels in the transgenic

342 overexpression lines were variable, but consistently 10 to 30 -fold above native MOS9 in both

343 wild-type and $t t 4-11$ backgrounds (Figures $3 \mathrm{~A}$ and S3A).

344

345 Effects of MOS9 on metabolism

346 Metabolite profiling was used to determine whether overexpression of MOS9 affected

347 plant metabolism, including the accumulation of flavonoids for which CHS activity is required.

348 Profiles were obtained from 5-day-old mos9-2, de35S::MOS9::YFP-1, de35S::MOS9::YFP-3,

$349 t 4-11$, and wild-type Col-0 seedlings by UPLC-ESI-MS. PCA analysis of the 502 metabolomic

350 features showed clear separation of the genotypes (Figure 4A), except for near superimposition

351 of the two genotypically-similar de35S::MOS9::YFP lines. Counter to expectations, the profile

352 for $\operatorname{mos} 9-2$ seedlings was not intermediate between wild-type Col-0 and the de35S::MOS9::YFP

353 lines. This suggests that the modest increase in MOS9 expression in $\operatorname{mos} 9-2$ is not sufficient to

354 mimic the effects on metabolism observed with overexpression in de35S::MOS9::YFP. 
A direct comparison of the profiles of de35S::MOS9::YFP and wild-type Col-0 is

356 illustrated in the feature distribution plot (Figure 4B). Of the 502 features generated by UPLC-

357 MS analysis, 26 were tightly correlated with the de35S::MOS9::YFP genotype, with eight at

358 higher levels and 18 at decreased levels relative to Col-0. After removal of redundant features

359 derived from adducts, the 26 features were associated with 18 distinct metabolites, 11 of which

360 could be assigned identities and were shown to fall into three distinct classes (Tables 2 and S3).

361

The largest group of metabolites correlated with overexpression of MOS9 consisted of

362 compounds derived from, or containing moieties derived from, phenylalanine. The most

363 substantial changes, from 4.1- to 12.1-fold, were observed for the flavonol, quercetin rhamnoside

364 (4.1-fold lower), and its bi- (12.1-fold lower) and triflavonoid (5.0-fold higher) forms (David et

365 al. 2014; Routaboul et al. 2006). The levels of these flavonoids were all slightly elevated in

$366 \operatorname{mos} 9-2$ and were entirely absent, as expected, in the CHS null mutant $t t 4-11$ (Bowerman et al.

367 2012). In addition to the MOS9 correlated features identified in the feature distribution plot, a

368 comparison of the flavonoids present in the metabolite profiles found small but significant

369 changes in the levels of other flavonol glycosides in the three MOS9 overexpression lines (Table

370 S4). These profiles of altered and overall reduced flavonoid accumulation are consistent with the

371 finding that MOS9 overexpression reduces CHS transcript levels, as well as the possibility that

372 interaction of MOS9 diverts CHS from its catalytic function. Additional statistically-significant

373 changes $(p<0.01)$ within the group of phenylalanine-derived compounds included increased (1.5-

374 to 2.5-fold) accumulation of three sinapoyl-containing compounds in the MOS9 overexpression

375 lines, one of which was also elevated in $t t 4-11$. Two additional phenylpropanoid-containing

376 compounds that are associated with defense functions exhibited statistically-significant changes

377 in the overexpression lines, with levels of 4-benzyloxybutyl glucosinolate elevated 2.8 fold, 
378 while syringaresinol glucoside was reduced 3.1-fold; these changes were somewhat smaller than

379 many of the others and for that reason perhaps less consistent in mos $9-2$ and $t t 4-11$. Taken

380 together, these observations provide further evidence that MOS 9 overexpression alters flux

381 across phenylpropanoid metabolism, a hallmark of disruption of its branch pathways, even if the

382 outcomes are not always easily predictable (Anderson et al. 2015; Ring et al. 2013).

383 A second group of metabolites associated with overexpression of MOS9 consisted of

384 arabidopsides, which are jasmonic acid precursors in the oxylipin family that have been

385 associated with defense (Griffiths 2015). Three of these compounds were present at

386 approximately 3 to 5-fold lower levels in the de35S::MOS9::YFP lines than in Col-0 (Table 2).

387 The third class was composed of compounds that could not be definitively identified, but

388 included four glycerolipids; these metabolites were present 2.5- to 6.4-fold lower levels than in

389 wild type plants. All of the compounds were also present at slightly reduced levels in $t t 4-11$,

390 although the changes were smaller and not all were statistically significant. Less clear were the

391 changes in $\operatorname{mos} 9-2$, which were overall higher relative to Col-0, but statistically significant in

392 only three cases. Overall, only the compounds with the largest changes in abundance in the

393 de35S::MOS9::YFP lines were also appreciably altered in mos 9-2. As above, the inconsistencies

394 between these lines likely reflect the comparatively small change in the level of mRNA

395 expression in the $\operatorname{mos} 9-2$ mutant line.

396

397 Effects of MOS9 overexpression on CHS transcript levels

398 To determine whether CHS expression was affected by overexpression of MOS9, CHS

399 transcript levels were assayed in roots of Col-0 wild type, mos9-2, and the two independent

400 de35S::MOS9::YFP lines in the Col-0 background. qRT-PCR analysis indicated that over- 
401 expression of MOS9 in seedling roots reduced the expression of CHS in a dose-dependent manner

402 in seedling roots. CHS transcript levels were significantly lower in two independent

403 de35S::MOS9::YFP lines, with an average expression of approximately $40 \%$ of wild-type levels

404 ( $\mathrm{p}<0.02)$, and appeared to be somewhat reduced in the overexpression mutant, mos 9-2, although

405 with $\mathrm{p}=0.109$ (Figures $3 \mathrm{~B}$ and $\mathrm{S} 3 \mathrm{~A}$ ). These results suggest that MOS9 influences CHS gene

406 expression at the level of transcription and/or mRNA stability, leading to a decrease in CHS

407 enzymatic activity, and thereby modulating the metabolite profile. The converse was not true,

408 however, with MOS9 transcripts at similar levels in the absence or presence of CHS (Figures 3A

409 and S3A)

410

\section{DISCUSSION}

412

This study endeavored to identify new interaction partners for the key flavonoid enzyme,

413 CHS, with the aim of adding to the catalog of components of an enzyme complex localized at the 414 cytoplasmic face of the ER and perhaps also in the nucleus (Winkel 2006). The interaction of 415 Arabidopsis CHS with the Atlg12530 gene product was initially deduced from a yeast two-

416 hybrid screen and subsequently supported by SPR analysis of recombinant proteins produced in

417 E. coli, indicating that the association does not rely on plant-specific post-translational

418 modifications. FLIM-FRET analysis of co-expressed fusion proteins further showed that the

419 interaction can occur in the near-native environment of Arabidopsis mesophyll protoplasts.

420 Overexpression of Atlg12530 resulted in changes in the seedling metabolome that overlapped

421 substantially with those observed in the $C H S$ null mutant, $t t 4-11$, consistent with a reduction in

422 CHS transcripts in the overexpression lines. These findings, together with evidence for its

423 subcellular localization, suggest that this newly-identified partner influences flavonoid 
424 metabolism and perhaps CHS gene expression through interaction with CHS protein in the 425 nucleus.

Remarkably, AT1g12530 has been independently described as one of 15 modifier of snc1

$427(\operatorname{mos})$ genes identified in a screen for suppressors of a constitutively-active allele of the NB-

428 LRR-encoding resistance (R) gene, sncl (suppressor of nprl-1 constitutive) (Monaghan et al.

429 2010). R genes function to recognize pathogen effectors and activate effector-triggered

430 immunity (ETI), a secondary defense mechanism that typically results in induction of the

431 hypersensitive response (Jones et al. 2016). The products of these 15 genes participate in a wide

432 range of fundamental cellular processes, from nucleo-cytoplasmic trafficking to RNA processing

433 and protein modification, all of which are also key components of the defense signaling network.

434 Xia at el. (2013) subsequently showed that MOS9 is essential for expression of both SNC1 and

435 the adjacent NB-LRR R gene, RPP4. The action of MOS9 appears to involve association with

436 ATXR7, a Set 1 class H3K4 histone methyltransferase that may also have a role in flowering

437 time determination (Yun et al. 2011). MOS9 and ATXR7 were initially copurified via

438 immunoprecipitation, but unlike the situation with MOS9 and CHS, additional interaction assays

439 were unsuccessful. This led the authors to propose that MOS9 and ATXR7 may not interact

440 directly, but rather constitute components of a larger complex that includes additional as-yet-to-

441 be identified proteins that together mediate the expression of key defense signaling genes.

442 The new evidence that MOS9 interacts with CHS and that overexpression is associated

443 with changes in $C H S$ mRNA levels and the profile of phenolics in roots suggests that MOS9 may

444 also play a role in controlling plant metabolism. One scenario is that MOS9 helps modulate CHS

445 gene expression in response to cellular CHS protein levels as part of a negative feedback loop.

446 The association of MOS9 with components of the $R$ gene expression network suggests that it 
447 could also serve to coordinate multiple cellular processes, including during plant defense.

448 Suppression of flavonoid gene expression and flavonoid levels is well known to accompany ETI,

449 ostensibly allowing plants to redirect carbon flow into defense-related phenylpropanoid

450 compounds such as scopoletin and lignin (Schenke et al. 2011). It may also promote expression

451 of defense-related genes such as late-response $P R-1$ and $P R-2$, which appear to be repressed

452 when flavonoids are present (Serrano et al. 2012). This suppression may involve histone

453 remodeling of several flavonoid structural and regulatory genes, including CHS (Schenke et al.

454 2014; Velanis et al. 2016; Zhou et al. 2017). One possibility is that the MOS9-CHS interaction

455 either directly or indirectly impacts epigenetic modifications associated with these genes,

456 analogous to its proposed role in control of $R$ gene expression.

457 It should be noted that the metabolite profiles of MOS9 overexpressing and CHS null

458 plants support the involvement of both proteins in additional, nonoverlapping, functions. Many

459 of the metabolites that distinguish the MOS9 overexpressing lines from wild type were also

460 altered in $t t 4-11$; overall, where there were substantial changes in the 35Sde::MOS9::YFP lines,

461 these were also observed in $\operatorname{mos} 9-2$ and $t t 4-11$ (Table 2). This includes a number of

462 phenylpropanoid and flavonoid compounds, consistent with CHS and MOS9 acting in, or on, the

463 same metabolic pathways. However, the opposite effects on 4-benzoyloxybutyl glucosinolate

464 levels suggest that MOS9 may also influences processes distinct from those stemming from

465 interaction with CHS. The apparent constitutive expression of MOS9 further suggests that, as

466 with its involvement in control of $R P P 4$ and $S N C 1$ gene expression, there are surely other factors

467 involved.

468

469 CONCLUSIONS 
471 localized protein, MOS9, may have uncovered a novel mechanism for controlling flavonoid

472 metabolism and linking this highly dynamic plant pathway with specific physiological functions,

473 including the defense response. Identifying additional components of the MOS9 interaction

474 network and elucidating the functions of the associated protein complexes will be key to shedding 475 further light on this intriguing system.

476

477 ACKNOWLEDGMENTS

478 The authors are indebted to William L. Crosby for the gift of the yeast two-hybrid library, to

479 Anna Pietraszewska-Bogiel and Dorus Gadella of the University of Amsterdam for providing the 480 expertise and facilities for the FLIM-FRET experiments, and to Kristi DeCourcy at Virginia 481 Tech for technical support with confocal microscopy.

482

483

\section{REFERENCES CITED}

484

485

486

487

488

489

490

491

492

Albers M, Kranz H, Kober I, Kaiser C, Klink M, Suckow J, Kern R, and Koegl M. 2005. Automated yeast two-hybrid screening for nuclear receptor-interacting proteins. Molecular and Cellular Proteomics 4:205-213. 10.1074/mcp.M400169-MCP200

Anderson NA, Bonawitz ND, Nyffeler K, and Chapple C. 2015. Loss of FERULATE 5HYDROXYLASE leads to mediator-dependent inhibition of soluble phenylpropanoid biosynthesis in Arabidopsis. Plant Physiology 169:1557-1567. 10.1104/pp.15.00294

Bechtold N, Ellis J, and Pelletier G. 1993. In planta Agrobacterium mediated gene transfer by infiltration of adult Arabidopsis thaliana plants. Comptes Rendus De l'Academie Des Sciences 316:1194-1199. 10.1007/978-3-642-79247-2_3 
493 Bowerman PA, Ramirez MV, Price MB, Helm RF, and Winkel BS. 2012. Analysis of T-DNA 494 alleles of flavonoid biosynthesis genes in Arabidopsis ecotype Columbia. BMC Res Notes 495 5:485. $10.1186 / 1756-0500-5-485$

496

497

498

499

500

501

502

503

504

505

506

507

508

509

510

511

512

513

514

515

Burbulis IE, and Winkel-Shirley B. 1999. Interactions among enzymes of the Arabidopsis flavonoid biosynthetic pathway. Proceedings of the National Academy of Sciences of the United States of America 96:12929-12934. 10.1073/pnas.96.22.12929

Crosby KC, Pietraszewska-Bogiel A, Gadella TWJ, and Winkel BSJ. 2011. Förster resonance energy transfer demonstrates a flavonoid metabolon in living plant cells that displays competitive interactions between enzymes. FEBS Letters 585:2193-2198. 10.1016/j.febslet.2011.05.066

David LC, Dechorgnat J, Berquin P, Routaboul JM, Debeaujon I, Daniel-Vedele F, and FerrarioMery S. 2014. Proanthocyanidin oxidation of Arabidopsis seeds is altered in mutant of the high-affinity nitrate transporter NRT2.7. Journal of Experimental Botany 65:885-893. $10.1093 / \mathrm{jxb} / \mathrm{ert} 481$

Earley KW, Haag JR, Pontes O, Opper K, Juehne T, Song KM, and Pikaard CS. 2006. Gatewaycompatible vectors for plant functional genomics and proteomics. Plant Journal 45:616629. 10.1111/j.1365-313X.2005.02617.x

Edwards K, Johnstone C, and Thompson C. 1991. A simple and rapid method for the preparation of plant genomic DNA for PCR analysis. Nucleic Acids Research 19:1349. 10.1093/nar/19.6.1349

Fromont-Racine M, Rain J-C, and Legrain P. 1997. Toward a functional analysis of the yeast genome through exhaustive two-hybrid screens. Nature Genetics 16:277-282. $10.1038 /$ ng0797-277 
516 Gayomba SR, Watkins JM, and Muday GK. 2016. Flavonols regulate plant growth and 517 development through regulation of auxin transport and cellular redox status. In: Yoshida 518 K, Cheynier V, and Quideau S, editors. Recent Advances in Polyphenol Research: John $519 \quad$ Wiley and Sons. p 143-170.

520 Griffiths G. 2015. Biosynthesis and analysis of plant oxylipins. Free Radical Research 49:565521 582. $10.3109 / 10715762.2014 .1000318$

522 Hrazdina G, and Jensen RA. 1992. Spatial organization of enzymes in plant metabolic pathways.

523

524

525

526

527

528

529

530

531

532

\section{3}

534

535

536 Annual Review of Plant Physiology and Plant Molecular Biology 43:241-267. 10.1146/annurev.pp.43.060192.001325

Hrazdina G, and Wagner GJ. 1985. Metabolic pathways as enzyme complexes: evidence for the synthesis of phenylpropanoids and flavonoids on membrane associated enzyme complexes. Archives of Biochemistry and Biophysics 237:88-100. 10.1016/0003-9861(85)90257-7

Jones JDG, Vance RE, and Dangl JL. 2016. Intracellular innate immune surveillance devices in plants and animals. Science 354. 10.1126/science.aaf6395

Karimi M, Inze D, and Depicker A. 2002. GATEWAY ${ }^{\mathrm{TM}}$ vectors for Agrobacterium-mediated plant transformation. Trends in Plant Science 7:193-195. 10.1016/S1360-1385(02)022513

Kohalmi SE, Reader LJV, Samach A, Nowak J, Haughn GW, and Crosby WL. 1998. Identification and characterization of protein interactions using the yeast 2-hybrid system. In: Gelvin SB, and Schiperoort RA, eds. Plant Molecular Biology Manual. Dordrecht, The Netherlands: Kluwer Academic Publishers, 1-30. 
537 Kubasek WL, Shirley BW, McKillop A, Goodman HM, Briggs W, and Ausubel FM. 1992.

538 Regulation of flavonoid biosynthetic genes in germinating Arabidopsis seedlings. Plant $539 \quad$ Cell 4:1229-1236. doi.org/10.1105/tpc.4.10.1229

540 Mercuri A, Sacchetti A, De Benedetti L, Schiva T, and Alberti S. 2001. Green fluorescent flowers. 541 Plant Science 161:961-968. 10.1016/s0168-9452(01)00497-6

542 Monaghan J, Germain H, Weihmann T, and Li X. 2010. Dissecting plant defence signal 543 transduction: modifiers of sncl in Arabidopsis. Canadian Journal of Plant Pathology $32: 35-42.10 .1080 / 07060661003621001$

545 Mouradov A, and Spangenberg G. 2014. Flavonoids: a metabolic network mediating plants 546 adaptation to their real estate. Frontiers in Plant Science 5:620. 10.3389/fpls.2014.00620

547 Owens DK, Alerding AB, Crosby KC, Bandara AB, Westwood JH, and Winkel BSJ. 2008a. 548 Functional analysis of a predicted flavonol synthase gene family in Arabidopsis. Plant Physiology 147:1046-1061. 10.1104/pp.108.117457

550 Owens DK, Crosby KC, Runac J, Howard BA, and Winkel BSJ. 2008b. Biochemical and genetic 551 characterization of Arabidopsis flavanone 3b-hydroxylase. Plant Physiology and 552 Biochemistry 46:833-843. 10.1016/j.plaphy.2008.06.004

553 Panche AN, Diwan AD, and Chandra SR. 2016. Flavonoids: an overview. Journal of Nutritional $554 \quad$ Science 5. 10.1017/jns.2016.41

555 Pelaz S, Gustafson-Brown C, Kohalmi SE, Crosby WL, and Yanofsky MF. 2001. APETALA1 556 and SEPALLATA3 interact to promote flower development. Plant Journal 26:385-394. 10.1046/j.1365-313X.2001.2641042.X

558 Pfaffl MW. 2001. A new mathematical model for relative quantification in real-time RT-PCR. 559 Nucleic Acids Research 29:6. 10.1093/nar/29.9.e45 
560 Ring L, Yeh SY, Huecherig S, Hoffmann T, Blanco-Portales R, Fouche M, Villatoro C, Denoyes

561 B, Monfort A, Caballero JL, Munoz-Blanco J, Gershenson J, and Schwab W. 2013.

562 Metabolic interaction between anthocyanin and lignin biosynthesis is associated with

563 peroxidase FaPRX27 in strawberry fruit. Plant Physiology 163:43-60.

$564 \quad 10.1104 /$ pp. 113.222778

565 Robic G, Lacorte C, and Miranda EA. 2009. Fluorometric quantification of green fluorescent 566 protein in tobacco leaf extracts. Analytical Biochemistry 392:8-11.

567 10.1016/j.ab.2009.05.016

568 Routaboul JM, Kerhoas L, Debeaujon I, Pourcel L, Caboche M, Einhorn J, and Lepiniec L. 2006.

$569 \quad$ Flavonoid diversity and biosynthesis in seed of Arabidopsis thaliana. Planta 224:96-107.

$570 \quad 10.1007 / \mathrm{s} 00425-005-0197-5$

571 Saslowsky D, Warek U, and Winkel BSJ. 2005. Nuclear localization of flavonoid metabolism in 572 Arabidopsis thaliana. Journal of Biological Chemistry 280:23735-23740.

573 10.1074/jbc.M413506200

574 Saslowsky D, and Winkel-Shirley B. 2001. Localization of flavonoid enzymes in Arabidopsis 575 roots. Plant Journal 27:37-48. 10.1046/j.1365-313x.2001.01073.x

576 Schenke D, Bottcher C, and Scheel D. 2011. Crosstalk between abiotic ultraviolet-B stress and 577

578 biotic (flg22) stress signalling in Arabidopsis prevents flavonol accumulation in favor of 579 pathogen defence compound production. Plant Cell and Environment 34:1849-1864.

580

581

582 10.1111/j.1365-3040.2011.02381.x

Schenke D, Cai DG, and Scheel D. 2014. Suppression of UV-B stress responses by flg22 is regulated at the chromatin level via histone modification. Plant Cell and Environment 37:1716-1721. 10.1111/pce.12283 
583 Serrano M, Kanehara K, Torres M, Yamada K, Tintor N, Kombrink E, Schulze-Lefert P, and Saijo 584 Y. 2012. Repression of sucrose/ultraviolet B light-induced flavonoid accumulation in 585 microbe-associated molecular pattern-triggered immunity in Arabidopsis. Plant $586 \quad$ Physiology 158:408-422. 10.1104/pp.111.183459

587 Shih CH, Chu H, Tang LK, Sakamoto W, Maekawa M, Chu IK, Wang M, and Lo C. 2008. $588 \quad$ Functional characterization of key structural genes in rice flavonoid biosynthesis. Planta 589 228:1043-1054. 10.1007/s00425-008-0806-1

590 Stafford HA. 1990. Flavonoid Metabolism. Boca Raton: CRC Press.

591 Stafford HA. 1991. Flavonoid evolution: an enzymic approach. Plant Physiology 96:680-685. 592 $10.1104 /$ pp. 96.3 .680

593 594 595 596 597 598 599 600 601 602 603 604 605

van Munster EB, and Gadella TWJ. 2005. Fluorescence lifetime imaging microscopy (FLIM). Microscopy Techniques. Berlin: Springer-Verlag Berlin, 143-175.

Velanis CN, Herzyk P, and Jenkins GI. 2016. Regulation of transcription by the Arabidopsis UVR8 photoreceptor involves a specific histone modification. Plant Molecular Biology 92:425-443. 10.1007/s11103-016-0522-3

Winkel-Shirley B. 1999. Evidence for enzyme complexes in the phenylpropanoid and flavonoid pathways. Physiologia Plantarum 107:142-149. 10.1034/j.1399-3054.1999.100119.x

Winkel-Shirley B. 2001. Flavonoid biosynthesis: a colorful model for genetics, biochemistry, cell biology and biotechnology. Plant Physiology 126:485-493. doi.org/10.1104/pp.126.2.485

Winkel-Shirley B. 2002. Biosynthesis of flavonoids and effects of stress. Current Opinion in Plant Biology 5:218-223. 10.1016/s1369-5266(02)00256-X

Winkel BSJ. 2004. Metabolic channeling in plants. Annual Review of Plant Biology 55:85-107. 10.1146/annurev.arplant.55.031903.141714 
606 Winkel BSJ. 2006. The biosynthesis of flavonoids. In: Grotewold E, ed. The Science of 607 Flavonoids. New York: Springer Science \& Business Media 71-95.

608 Winkel BSJ. 2018. The subtleties of subcellular distribution: pointing the way to underexplored 609 functions for flavonoid enzymes and endproducts In: Schlosser C, and Halbwirth H, eds. 610 Recent Advances in Polyphenol Research: Wiley Inc., invited article, in press.

611 Xia ST, Cheng YT, Huang S, Win J, Soards A, Jinn TL, Jones JDG, Kamoun S, Chen S, Zhang 612 YL, and Li X. 2013. Regulation of transcription of nucleotide-binding leucine-rich repeatencoding genes SNC1 and RPP4 via H3K4 trimethylation. Plant Physiology 162:16941705. 10.1104/pp.113.214551

615

Yoo SD, Cho YH, and Sheen J. 2007. Arabidopsis mesophyll protoplasts: a versatile cell system for transient gene expression analysis. Nature Protocols 2:1565-1572.

621 Zhou Z, Schenke D, Miao Y, and Cai DG. 2017. Investigation of the crosstalk between the flg22 622

623 and the UV-B-induced flavonol pathway in Arabidopsis thaliana seedlings. Plant Cell and

624 Zimmermann P, Hirsch-Hoffmann M, Hennig L, and Gruissem W. 2004. GENEVESTIGATOR. 625 626 Arabidopsis microarray database and analysis toolbox. Plant Physiology 136:2621-2632. doi.org/10.1104/pp.104.046367

627 


\section{Figure 1}

Confirmation of the interaction of CHS with the At1g12530 protein.

A) SPR analysis. Purified, recombinant CHS was immobilized on an SPR chip and purified, recombinant MOS9 passed over at the indicated concentrations. Each binding plot is shown as micro response units (mRIU) over time and represents an average of three separate injections of MOS9. B) FLIM-FRET analysis of At1g12530-SCFP3A and CHS-SYFP2 in Arabidopsis mesophyll protoplasts. N-terminal fusion constructs of either At1g12530 or CHS with cyan fluorescent protein and yellow fluorescent protein (respectively) were generated and used to transform living protoplasts. Fluorescence lifetime of At1g12530-SCFP3A alone was compared with its fluorescent lifetime in the presence of CHS-YFP2. Protoplasts are pseudo-colored to represent measured $\tau$ values. E-values for phase-shifts were calculated using $n>20$ protoplasts (Table 1 ). 
A.

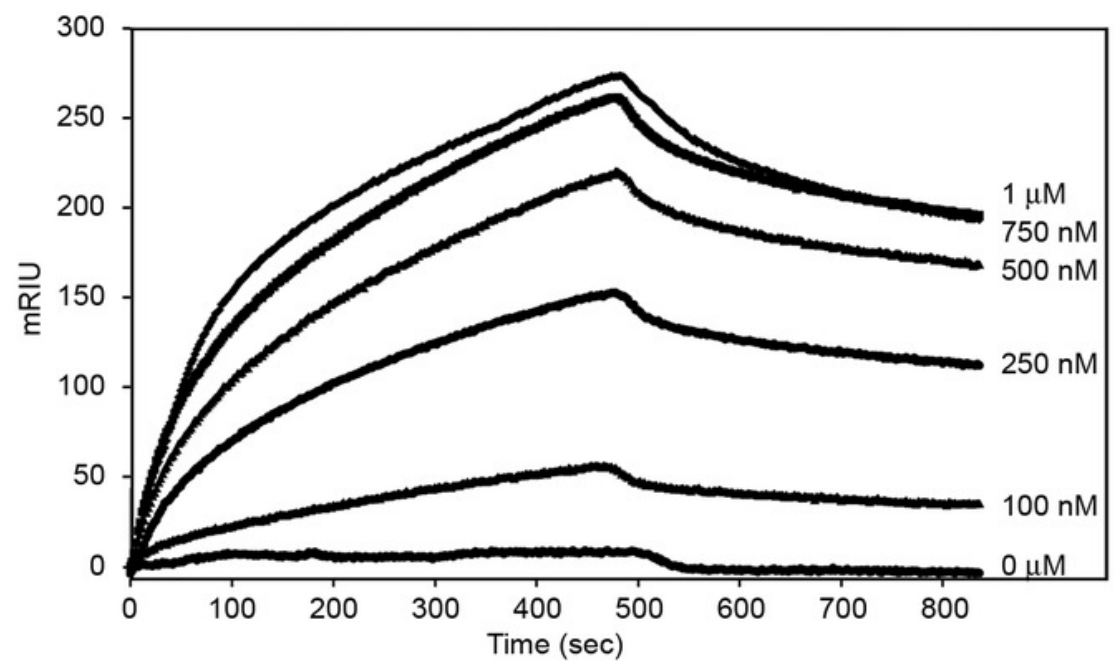

At1g12530-scfp3a

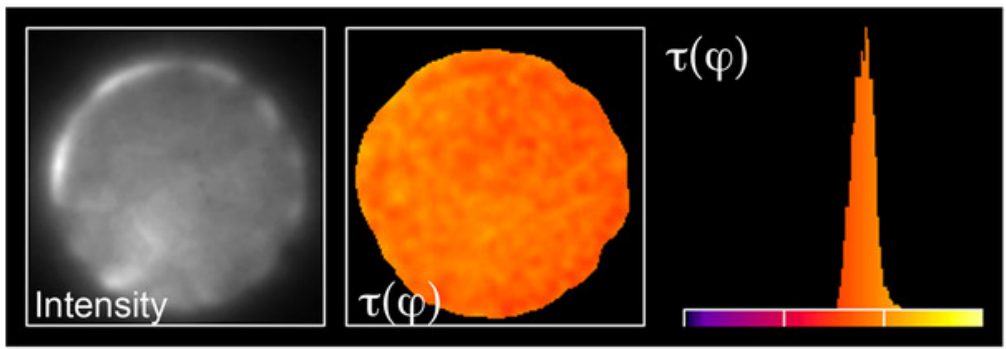

$\tau_{\mathrm{D}}=2.4 \mathrm{~ns}$

B.

Intensity
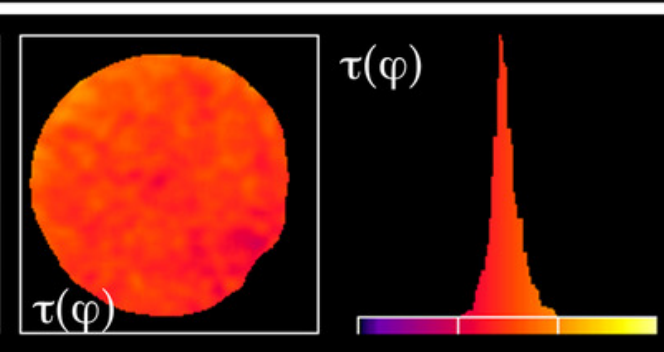

$\tau_{\mathrm{DA}}=2.2 \mathrm{~ns}$ $E=6.5 \%$

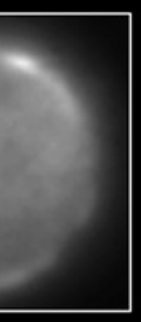

$\tau(\varphi)$ 


\section{Figure 2}

Localization of MOS9 in wild-type and CHS-deficient roots.

MOS9-YFP fusion proteins were expressed from the de35S promoter in stably-transformed wild-type Col-0 (A) and tt4-11 plants (B). The root elongation zones of five-day-old transgenic and Col-0 control (C) seedlings were examined by confocal laser scanning microscopy. All three images were taken under identical conditions and represent individual optical slices from stacks taken in the z-direction.

A.

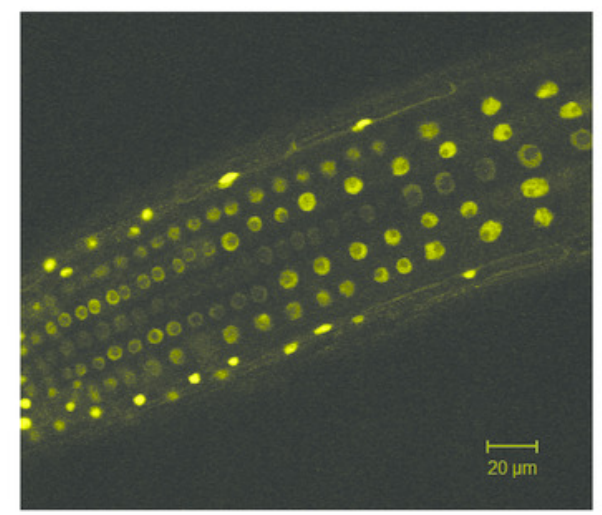

35S::MOS9::YFP in Col-0
B.

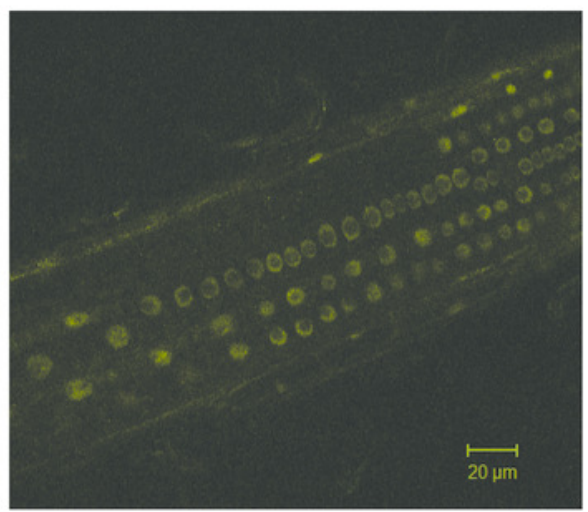

35S::MOS9::YFP in tt4-11
C.

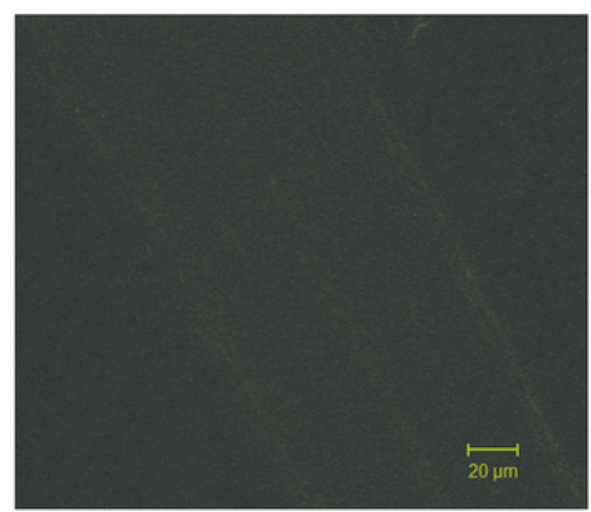

Col-0 
Figure 3 (on next page)

Analysis of MOS9 and CHS expression in roots of 5-day-old MOS9 overexpression lines.

Transcript levels were analyzed by Real-time PCR and normalized to the endogenous control gene, GAPDH. Error bars represent the standard error of the mean. Asterisks indicate a $\mathrm{p}$ value $<0.02$ from a two-tailed paired student's T-test for expression of $\mathrm{CHS}$ relative to wild type. WT = wild type (Col-0). 
A.

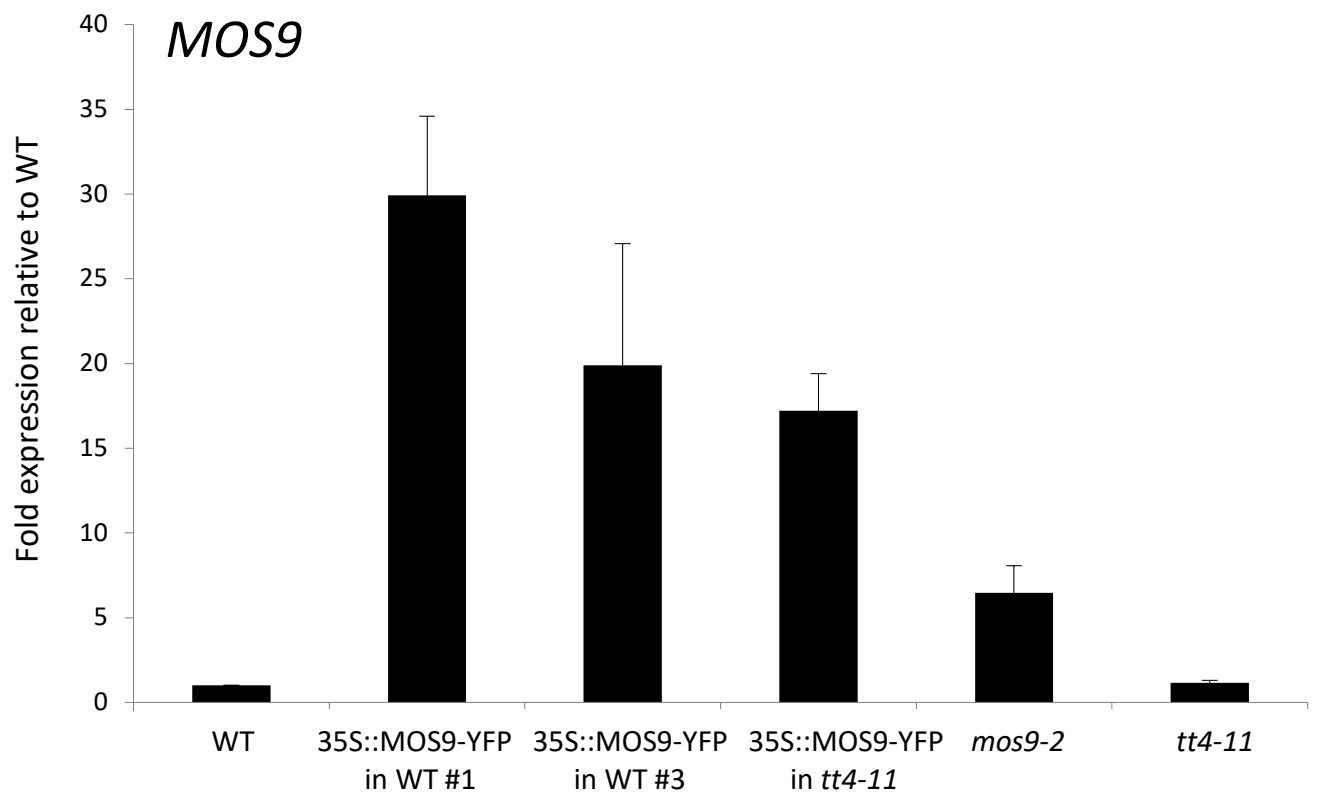

B.

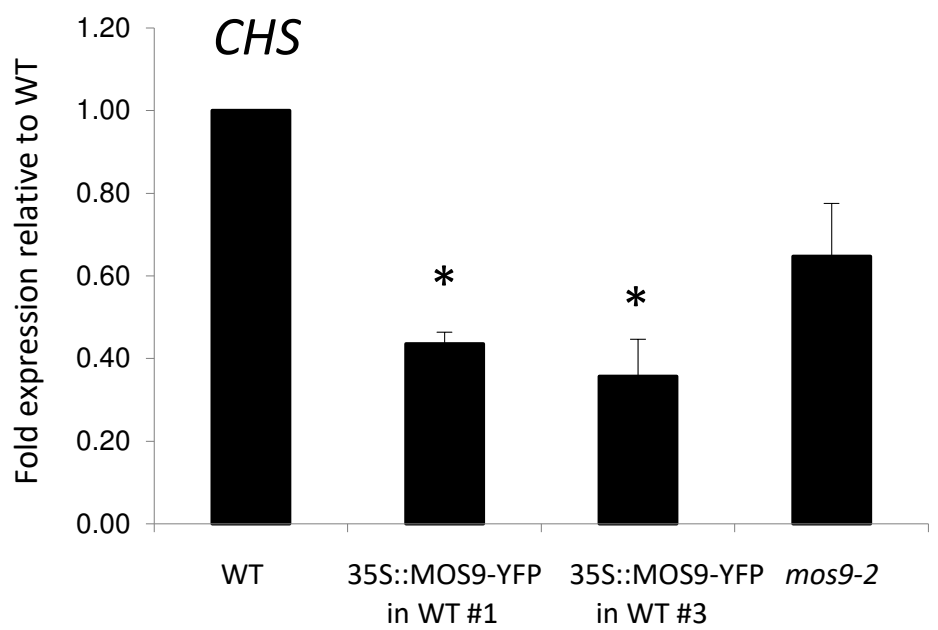

PeerJ reviewing PDF | (2018:03:26436:1:1:NEW 5 Aug 2018) 


\section{Figure 4}

Metabolite profiling of de35S::MOS9::YFP, mos9-2, tt4-11, and Col seedlings

A) Principal component analysis (PCA) of UPLC-MS profiles for four biological replicates of each genetic background. B) S-plot distribution of features between de35S::MOS9::YFP and Col-0 seedlings. Features shown in red were found to be associated with the MOS9 ectopic expression and were selected for further analysis. 


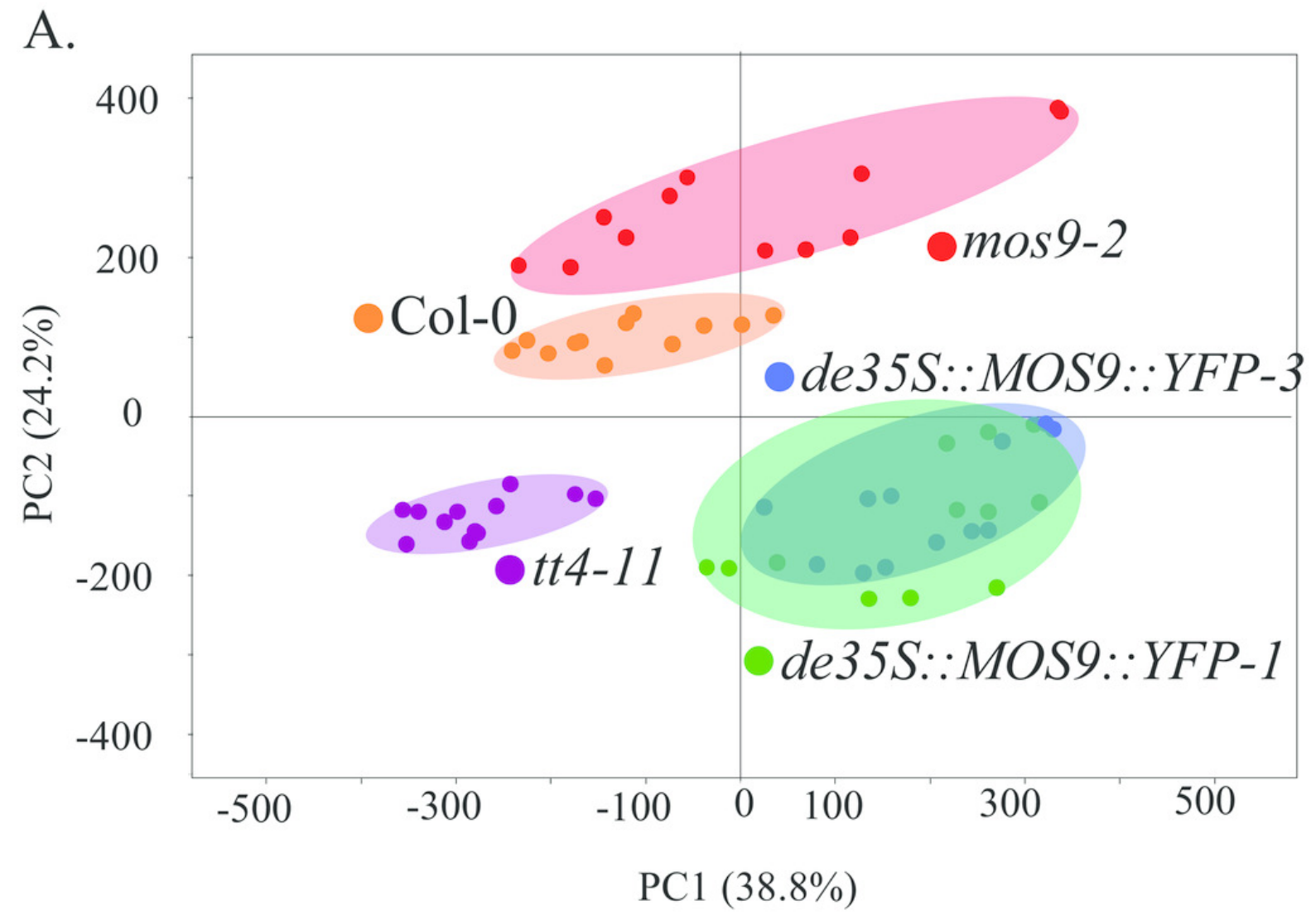

B.

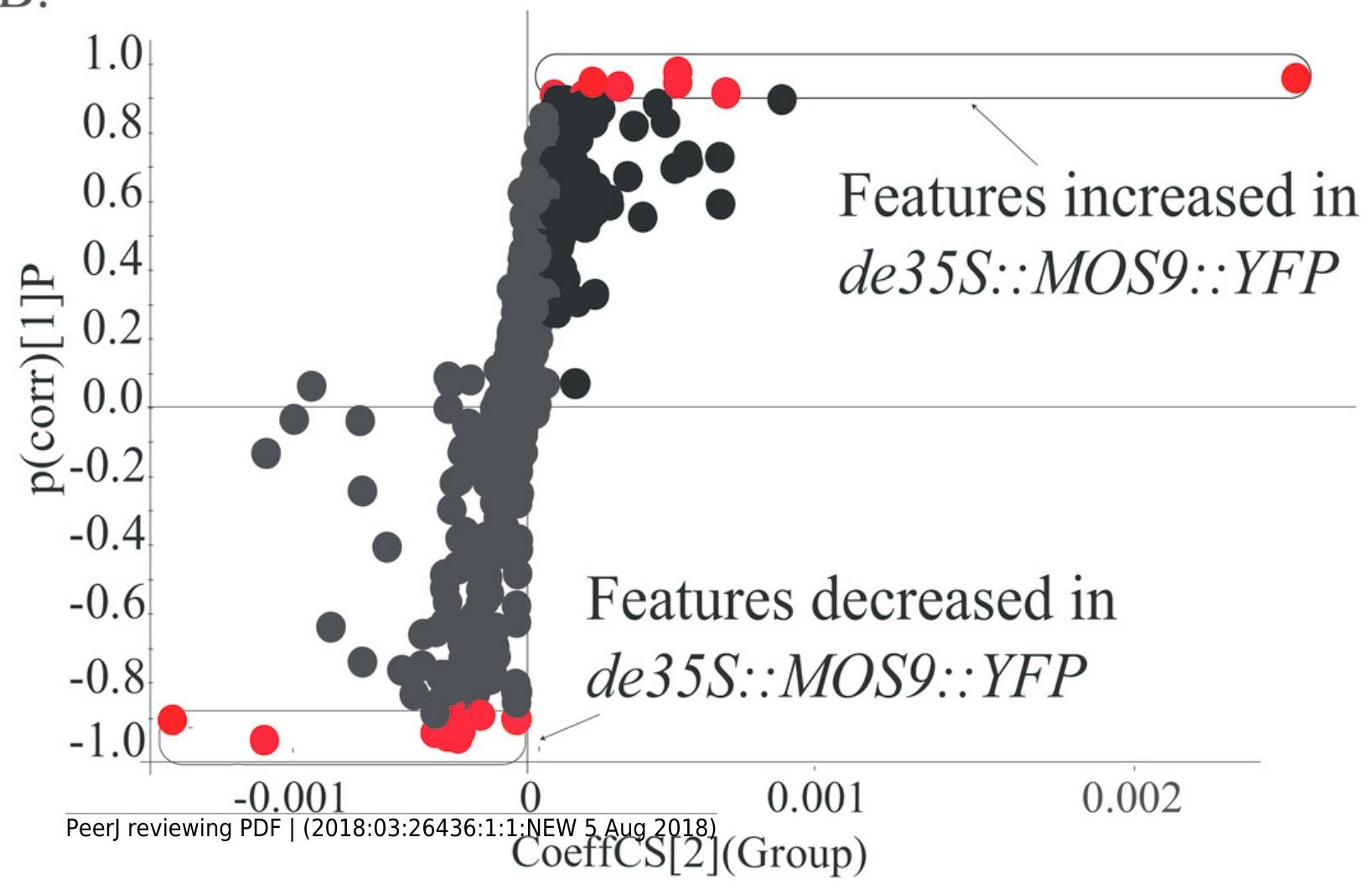




\section{Table 1 (on next page)}

FLIM-FRET parameters for analysis of the CHS-MOS9 interaction in Arabidopsis protoplasts. 
1 Table 1. FLIM-FRET parameters for analysis of the CHS-MOS9 interaction in Arabidopsis

2 protoplasts $^{\mathrm{a}}$

3

10

11

${ }^{a}$ Results above and below the dotted line are from independent experiments.

4 Donor Acceptor

5 MOS9-scfp3a

6 MOS9-scfp3a

7 MOS9-scfp3a

8 MOS9-scfp3a -

9 MOS9-scfp3a syfp2

- 24

CHS-syfp2 25

21

12
11

11

1

$\tau(\varphi) \mathrm{ns} \pm \mathrm{S} . \mathrm{D}$

$2.36 \pm 0.07$

$2.21 \pm 0.08$

$2.29 \pm 0.13$

$2.40 \pm 0.05$

$2.41 \pm 0.03$

$\tau(m) \mathrm{ns}$

$3.27 \pm 0.05$

$3.24 \pm 0.08 \quad 6.5 \%$

$3.37 \pm 0.07$

$3.26 \pm 0.03 \quad-$

$3.17 \pm 0.05 \quad-0.5 \%$ 


\section{Table 2 (on next page)}

Metabolites with altered levels in de35S::MOS9::YFP, mos9-2, and tt4-11 seedlings relative to wild-type Col-0. 
Table 2. Metabolites with altered levels in de35S::MOS9::YFP, mos9-2, and $t t 4$ seedlings relative to wild-type Col-0

Putative Identity ${ }^{\mathrm{a}}$

Retention

Observed

Time

Mass (Adduct)

de35S::

MOS9::YFP

Ratio of Change (p-value) $)^{b, c}$

\section{Phenylalanine Derivatives}

$\operatorname{mos} 9-2$

$t t 4-11$

Sinapoyl-glutamate $\quad 4.52$

$N, N$-Bis(sinapoyl)-spermidine $\quad 5.62$

1,2-Di-O-sinapoyl- $\beta$-glucose $\quad 6.40$

4-Benzoyloxybutyl-glucosinolate $\quad 5.77$

Quercetin-Rhamnose

$\begin{array}{ll}\text { Monomer } & 5.92 \\ \text { Biflavonoid } & 5.23 \\ \text { Triflavonoid } & 3.77 \\ \text { Syringaresinol glucoside } & 6.00\end{array}$

II. Jasmonic Acid Precursors/Oxylipins

Arabidopside A (isomer a) $\quad 11.36$

Arabidopside A (isomer b) $\quad 11.70$

Arabidopside $\mathrm{C}$

10.74

$819.4523\left(\left[\mathrm{M}-\mathrm{H}+\mathrm{HCO}_{2} \mathrm{H}\right]^{-}\right)$ $819.4523\left(\left[\mathrm{M}-\mathrm{H}+\mathrm{HCO}_{2} \mathrm{H}\right]^{-}\right)$

$981.5046\left(\left[\mathrm{M}-\mathrm{H}+\mathrm{HCO}_{2} \mathrm{H}\right]^{-}\right)$

$-2.9(<0.01)$

$-2.9(<0.01)$

\section{As-yet unidentified}

UK Glycerolipid 1

UK Glycerolipid 2

UK Glycerolipid 3

UK Glycerolipid 4

9.91
10.16
10.22
10.38
4.35
5.61
10.41

$855.4731\left(\left[\mathrm{M}-\mathrm{H}+\mathrm{HCO}_{2} \mathrm{H}\right]^{-}\right)$

$855.4741\left(\left[\mathrm{M}-\mathrm{H}+\mathrm{HCO}_{2} \mathrm{H}\right]^{-}\right)$

$957.4696\left(\left[\mathrm{M}-\mathrm{H}+\mathrm{HCO}_{2} \mathrm{H}\right]^{-}\right)$

$767.3841\left(\left[\mathrm{M}-\mathrm{H}+\mathrm{HCO}_{2} \mathrm{H}\right]^{-}\right)$

$307.0847\left([\mathrm{M}-\mathrm{H}]^{-}\right)$

$595.2021\left([\mathrm{M}-\mathrm{H}]^{-}\right)$

UK6

10.41

$783.3788\left(\left[\mathrm{M}-\mathrm{H}+\mathrm{HCO}_{2} \mathrm{H}\right]^{-}\right)$

$-3.1(<0.01)$
$-3.2(<0.01)$
$-2.6(<0.01)$
$-3.2(<0.01)$
$-6.4(<0.01)$
$-5.1(<0.01)$
$-2.5(<0.01)$

$$
\begin{array}{r}
1.1(0.43) \\
1.1(0.53) \\
1.1(0.02) \\
\mathbf{1 . 5}(<\mathbf{0 . 0 1 )}
\end{array}
$$

$2.1(<0.01)$

$1.1(0.33)$

$1.2(0.29)$

$-1.4(<0.01)$

$\begin{array}{rr}-4.1(<0.01) & -1.2(0.03) \\ -12.1(<0.01) & -1.5(<0.01) \\ -\mathbf{5 . 0}(<\mathbf{0 . 0 1}) & -1.8(<\mathbf{0 . 0 1}) \\ \mathbf{- 3 . 1}(<\mathbf{0 . 0 1}) & \mathbf{1 . 5}(<\mathbf{0 . 0 1})\end{array}$

n.d.

n.d.

n.d.

$-1.5(<0.01)$

${ }^{a}$ UK, unknown; additional information on metabolite identification is presented in Table S1.

${ }^{\mathrm{b}}$ Statistically-significant values are highlighted in bold type.

\begin{tabular}{|c|c|}
\hline $1.4(0.07)$ & $-1.0(0.85)$ \\
\hline $1.4(<0.01)$ & $-1.1(0.66)$ \\
\hline $1.2(0.22)$ & $-1.2(0.12)$ \\
\hline $1.0(0.72)$ & $-1.2(0.10)$ \\
\hline $1.9(<0.01)$ & $-1.7(<0.01)$ \\
\hline $1.7(<0.01)$ & $-1.4(<0.01)$ \\
\hline $1.1(0.89)$ & $-1.3(0.01)$ \\
\hline
\end{tabular}

${ }^{c}$ n.d. $=$ not detected

$$
\begin{array}{cc}
1.2(0.35) & -1.1(0.25) \\
1.3(0.09) & -1.1(0.32) \\
1.3(0.33) & -\mathbf{1 . 4}(<\mathbf{0 . 0 1})
\end{array}
$$

